Public Abstract

First Name:Amit

Middle Name:

Last Name:Kataria

Adviser's First Name:Chengshan

Adviser's Last Name:Xiao

Co-Adviser's First Name:

Co-Adviser's Last Name:

Graduation Term:FS 2007

Department:Electrical Engineering

Degree:MS

\title{
Title: Cognitive Radios - Spectrum Sensing Issues
}

Today wireless networks are characterized by fixed spectrum assignment policy. With ever increasing demand for frequency spectrum and limited resource availability FCC decided to make a paradigm shift by allowing more and more number of unlicensed users to transmit their signals in licensed bands so as to efficiently utilize the available spectrum. The motivating factor behind this decision was the findings in a report by Spectrum Policy Task Force, in which vast temporal and geographic variations in spectrum usage were found ranging from $15 \%$ to $85 \%$. FCC was now open to new approaches for efficient spectrum sharing techniques with unlicensed users; one of them is Cognitive Radios.

Cognitive Radio can smartly senses and adapts with the changing environment by altering its transmitting parameters, such as modulation, frequency, frame format etc. The main challenges with cognitive radios are that it should not interfere with the licensed users and should vacate the band when required. For this it should sense the signals faster. For this purpose various detection schemes like energy detector, matched filter and cyclostationary feature detector are discussed in this report and performance evaluation of these is calculated. Besides this a performance evaluation is done between cooperative and non cooperative spectrum sensing schemes which uses Amplify-and-Forward algorithm is also discussed. All simulations are done in MATLAB. 